\title{
Direct Observation of the Orientation of Urea Molecules at Charged Interfaces
}

\author{
Carolyn J. Moll, Jan Versluis, and Huib J. Bakker*
}

Cite This: J. Phys. Chem. Lett. 2021, 12, 10823-10828

Read Online

ABSTRACT: Dissolving urea into water induces special solvation properties that play a crucial role in many biological processes. Here we probe the properties of urea molecules at charged aqueous interfaces using heterodyne-detected vibrational sum-frequency generation (HD-VSFG) spectroscopy. We find that at the neat water/air interface urea molecules do not yield a significant sum-frequency generation signal. However, upon the addition of ionic surfactants, we observe two vibrational bands at 1660 and $1590 \mathrm{~cm}^{-1}$ in the HD-VSFG spectrum, assigned to mixed bands of the $\mathrm{C}=\mathrm{O}$ stretch and $\mathrm{NH}_{2}$ bend vibrations of urea. The orientation of the urea molecules depends on the sign of the charge localized at surface and closely follows the orientation of the neighboring water molecules. We demonstrate that urea is an excellent probe of the local electric field at aqueous interfaces.

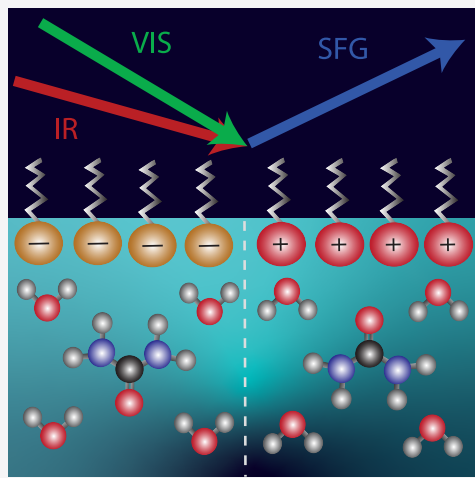

A queous solutions of urea show many striking properties, like the inhibition of micellar aggregation of surfactants and the enhancement of the solubility of hydrocarbons. ${ }^{1-4}$ Furthermore, urea plays a key role in many biological processes, of which the best known is the denaturation of proteins. $^{5-8}$ Due to these remarkable properties, aqueous solutions of urea have been the subject of many experimental and theoretical studies over the past decades. ${ }^{1,9-13}$ A great deal of these studies focused on the question to what extent the hydrogen bond network of water gets perturbed by adding urea molecules. The answer to this question is highly important to understanding the denaturation process of proteins by urea. In previous nuclear magnetic resonance (NMR) and molecular dynamics (MD) simulations studies, it has been proposed that urea breaks the hydrogen bond structure of water and thereby enhances the solvation of the hydrophobic groups of the protein. ${ }^{1-13}$ However, the results of recent pump-probe infrared (IR) measurements, nuclear magnetic resonance (NMR), and terahertz $(\mathrm{THz})$ absorption spectroscopy studies contradict such a hypothesis by showing that urea has no significant effect on the hydrogen bond network of water. ${ }^{2,14,15}$ These latter studies indicate that the protein denaturation is driven by a direct mechanism in which the urea molecules interact with the amide groups of the backbone of the protein. ${ }^{14} \mathrm{Up}$ to now, primarily the properties of urea in the bulk of aqueous solutions have been studied, and little work has been done addressing the properties of urea at aqueous surfaces. Recently surface tension measurements supported by MD-simulations showed that the surface tension increases proportionally with the urea concentration. ${ }^{16-20}$ Previous vibrational sum-frequency generation (VSFG) spec- troscopy studies of solutions containing $\alpha$-lactalbumin and urea showed that the orientation of the amide groups of the protein at the surface is independent from that of the urea molecules. This observation suggests that there are no strong direct interactions between the amide groups of the protein and the urea molecules, and points at an indirect denaturation process of the protein. ${ }^{21,22}$ In this work, we perform heterodyne-detected VSFG (HD-VSFG) measurements to investigate the molecular response of urea molecules at charged aqueous interfaces, by measuring the effect of the surface charge on the vibrational response of urea. Our results demonstrate that urea orients within the hydrogen-bonded network of water and that its absolute orientation is dependent on the sign of the charged surface.

In Figure 1, we present the experimental $\operatorname{Im}\left[\chi^{(2)}\right]$ spectra of neat water (dark blue) and aqueous urea solutions with a concentration of $1 \mathrm{M}$ (dark cyan) and $6 \mathrm{M}$ (dark magenta) in the frequency region of $1550-1750 \mathrm{~cm}^{-1}$. For pure water, we observe a symmetric positive peak centered at $1656 \mathrm{~cm}^{-1}$ that we assign to the water bending mode. The observed spectrum of the neat water surface agrees well with the results of previous HD-VSFG studies. ${ }^{23-25}$ The $\operatorname{Im}\left[\chi^{(2)}\right]$ spectra of the aqueous urea solutions with concentrations of 1 and $6 \mathrm{M}$ both show a symmetric peak at $1656 \mathrm{~cm}^{-1}$, identical with what is

Received: September 13, 2021

Accepted: October 26, 2021

Published: November 2, 2021 


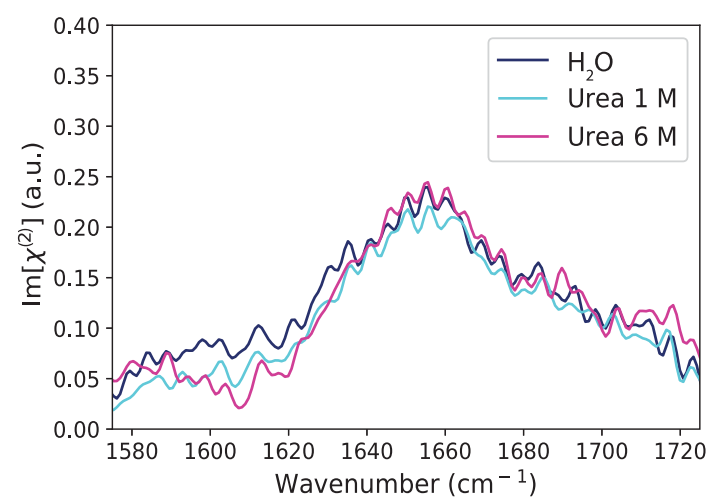

Figure 1. $\operatorname{Im}\left[\chi^{(2)}\right]$ spectra of the neat water surface (dark blue) and aqueous urea solutions with a concentration of $1 \mathrm{M}$ (dark cyan) and 6 $\mathrm{M}$ (dark magenta) within the frequency region of $1550-1750 \mathrm{~cm}^{-1}$.

observed for the neat water surface, and that we assign to the water bending mode. This observation suggests that even at a high urea concentration of $6 \mathrm{M}$ no characteristic vibrational modes of urea are observed. This implies that urea is either not located in the vicinity of the surface and/or does not show a net orientation near an uncharged water surface. This observation is consistent with the results of VSFG experiments in the $\mathrm{OH}$ and $\mathrm{NH}$ stretch vibrational region by Stratzdaite et al. $^{21}$

Figure $2 \mathrm{a}$ shows the $\operatorname{Im}\left[\chi^{(2)}\right]$ spectrum of an aqueous sodium dodecyl sulfate (SDS) solution (dotted magenta) with a concentration of $2 \mathrm{mM}$ and the $\operatorname{Im}\left[\chi^{(2)}\right]$ spectrum of the same solution of SDS with $1 \mathrm{M}$ of urea added (solid dark magenta). We used a concentration of $2 \mathrm{mM}$ of SDS. With this concentration, the expected phase distortion effects are expected to be negligible in the HD-VSFG spectra, as the electrical double layer (EDL) has a thickness $<10 \mathrm{~nm}$ for ionic strengths $>1 \mathrm{mM}^{26-28}$ To verify this notion, we modeled the expected phase distortion using the Poisson-Boltzmann equation for a solution with an ionic strength of $2 \mathrm{mM}$. We find the phase change to be less than 10 degrees (see Figure $\mathrm{S} 1$ ), which means that this change is within the experimental phase uncertainty. The $\operatorname{Im}\left[\chi^{(2)}\right]$ spectrum of an aqueous $2 \mathrm{mM}$ SDS solution shows a negative and a positive peak centered at 1626 and $1656 \mathrm{~cm}^{-1}$, which we assign to the dipolar and quadrupolar response of the water bending mode, respectively. ${ }^{25}$ The negative sign of the dipolar contribution implies that the water molecules have a net orientation with their hydrogen atoms toward the surface, as a result of the negative charge of the surface imposed by the SDS surfactants. ${ }^{25}$ In Figure $2 \mathrm{c}$, we present the $\operatorname{Im}\left[\chi^{(2)}\right]$ spectrum of an aqueous dodecyltrimethylammonium bromide (DTAB) solution (dotted cyan) with a concentration of $2 \mathrm{mM}$ and the $\operatorname{Im}\left[\chi^{(2)}\right]$ spectrum of the same solution of DTAB containing $1 \mathrm{M}$ of urea (solid dark cyan). The $\operatorname{Im}\left[\chi^{(2)}\right]$ of DTAB shows a single positive peak centered at $1640 \mathrm{~cm}^{-1}$ that in comparison to the band observed in the $\operatorname{Im}\left[\chi^{(2)}\right]$ spectrum of neat water shown in Figure 1 is enhanced, broadened, and shifted $\sim 10$ wavenumbers to lower frequencies. This broad band can be separated in two frequency components, centered at 1626 and $1656 \mathrm{~cm}^{-1}$, and assigned to the dipolar and quadrupolar responses of the water bending mode, respectively. ${ }^{25}$ In the case of the DTAB solution, the dipolar contribution has a positive $\operatorname{Im}\left[\chi^{(2)}\right]$ response, indicating a net orientation of the water molecules with their hydrogen atoms toward the bulk, as

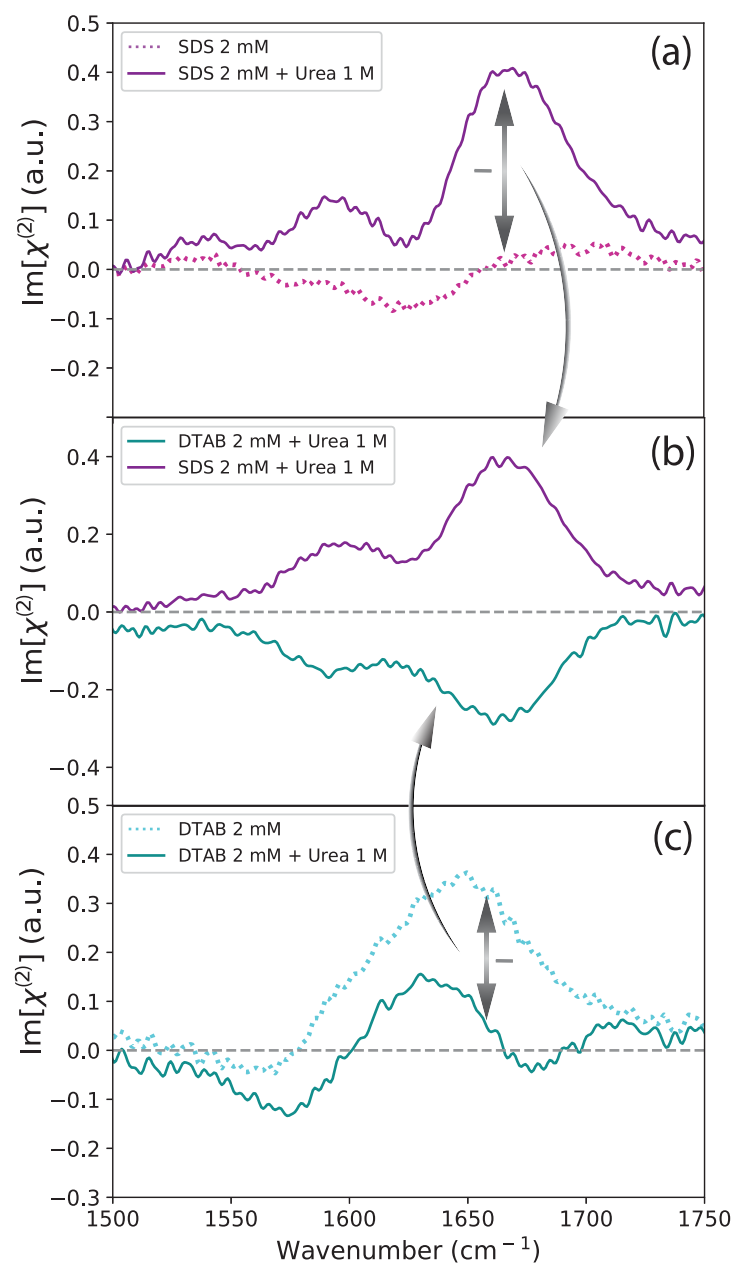

Figure 2. $\operatorname{Im}\left[\chi^{(2)}\right]$ spectra of aqueous solutions of different surfactants and different concentrations of urea. (a) $\operatorname{Im}\left[\chi^{(2)}\right]$ spectra of a solution with $2 \mathrm{mM}$ SDS (dotted magenta), and of a solution with $2 \mathrm{mM}$ SDS and $1 \mathrm{M}$ urea (solid dark magenta). (b) $\operatorname{Im}\left[\chi^{(2)}\right]$ spectra of aqueous solutions containing $1 \mathrm{M}$ urea and either $2 \mathrm{mM}$ SDS or $2 \mathrm{mM}$ DTAB, after subtraction of the response of the water bending mode. (c) $\operatorname{Im}\left[\chi^{(2)}\right]$ spectra of a solution with $2 \mathrm{mM} \mathrm{DTAB}$ (dotted cyan) and of a solution with $2 \mathrm{mM} \mathrm{DTAB}$ and $1 \mathrm{M}$ of urea (solid dark cyan).

a result of the positive charge at the surface created by the positively charged DTAB surfactants.

The presence of $1 \mathrm{M}$ urea leads to a significant change of the $\operatorname{Im}\left[\chi^{(2)}\right]$ spectra of the aqueous solutions of SDS and DTAB, as shown in parts a and $c$ of Figure 2. The resulting spectra (solid dark magenta and solid dark cyan) look very different from the $\operatorname{Im}\left[\chi^{(2)}\right]$ of the aqueous solutions containing only SDS (dotted magenta) and only DTAB (dotted cyan). In Figure $2 b$, we have subtracted the $\operatorname{Im}\left[\chi^{(2)}\right]$ response of the water bending mode from that of the solution containing 2 $\mathrm{mM}$ SDS and $1 \mathrm{M}$ urea and from that of the solution containing $2 \mathrm{mM} \mathrm{DTAB}$ and $1 \mathrm{M}$ urea. The difference spectra show two distinct vibrational bands centered at 1590 and 1660 $\mathrm{cm}^{-1}$ that we assign to mixed bands of the $\mathrm{C}=\mathrm{O}$ stretch and $\mathrm{NH}_{2}$ bend vibrations of the urea molecule. The mode at 1590 $\mathrm{cm}^{-1}$ is dominated by the $\mathrm{NH}_{2}$ bending vibrations, while the band centered at $1660 \mathrm{~cm}^{-1}$ is dominated by the $\mathrm{C}=\mathrm{O}$ stretch vibration. ${ }^{29,30}$ The band centered at $1660 \mathrm{~cm}^{-1}$ has a higher absolute amplitude than the peak at $1590 \mathrm{~cm}^{-1}$, which can be explained by the larger transition dipole moment of the $\mathrm{C}=\mathrm{O}$ stretch vibration. To confirm the above assignment of the 

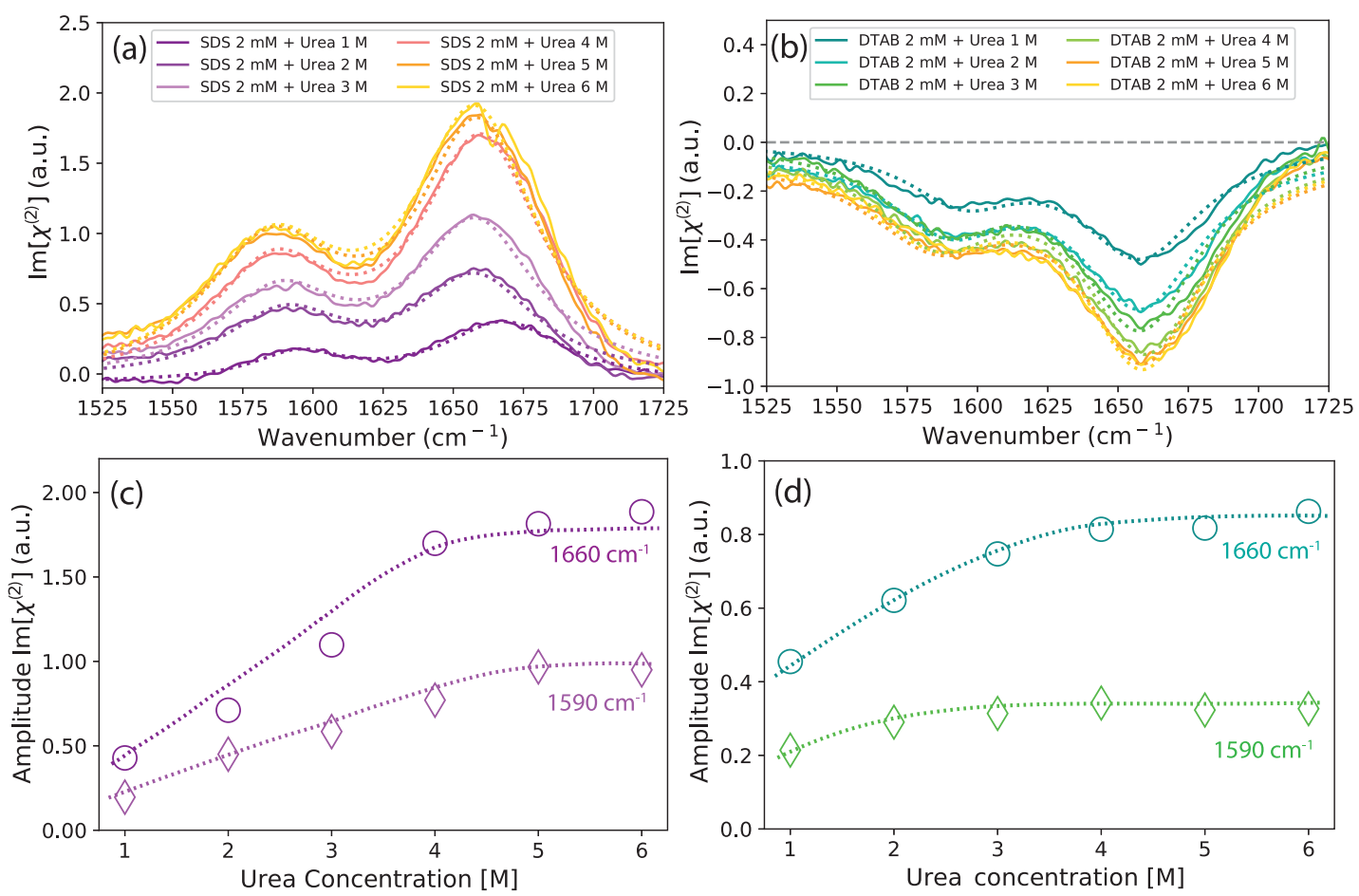

Figure 3. $\operatorname{Im}\left[\chi^{(2)}\right]$ spectra of aqueous solutions containing different concentrations of urea, ranging from 1 to $6 \mathrm{M}$, and (a) $2 \mathrm{mM}$ SDS and (b) 2 $\mathrm{mM}$ DTAB. For all measurements the signature of the water bending mode is subtracted from the $\operatorname{Im}\left[\chi^{(2)}\right]$ spectrum. Parts $\mathrm{c}$ and $\mathrm{d}$ show the absolute amplitude of the bands centered at 1660 and $1590 \mathrm{~cm}^{-1}$ extracted from the fitting procedure as a function of the concentration of urea for (c) a solution containing $2 \mathrm{mM}$ SDS and (d) a solution containing $2 \mathrm{mM}$ DTAB. The dotted lines are guides to the eye.

vibrational modes of urea, we also performed measurements with deuterated urea dissolved in $\mathrm{D}_{2} \mathrm{O}$. In deuterated urea, the $\mathrm{NH}_{2}$ groups of urea are exchanged by $\mathrm{ND}_{2}$ groups, leading to a significant redshift of the frequency of the bending vibration of these groups and a decoupling from the $\mathrm{C}=\mathrm{O}$ stretch vibration. Additionally, the bending vibration of $\mathrm{D}_{2} \mathrm{O}$ is shifted to lower frequencies with respect to the bending mode of $\mathrm{H}_{2} \mathrm{O}$. For this system, we observe only a band at $1600 \mathrm{~cm}^{-1}$ that can be assigned to the nearly pure $\mathrm{C}=\mathrm{O}$ stretching mode of the deuterated urea molecule (see Figure S2), thus confirming the assignment of the 1590 and $1660 \mathrm{~cm}^{-1}$ to bands of mixed $\mathrm{C}=$ $\mathrm{O}$ stretch and $\mathrm{NH}_{2}$ bending vibration character. ${ }^{29,31}$

Interestingly, the signs of the 1590 and $1660 \mathrm{~cm}^{-1}$ bands in the $\operatorname{Im}\left[\chi^{(2)}\right]$ spectra of Figure $2 \mathrm{~b}$ are governed by the sign of the charge at the surface. For a solution with $2 \mathrm{mM}$ SDS and 1 $\mathrm{M}$ urea, the two vibrational modes have a positive sign, which implies that the urea molecules near the surface orient with their $\mathrm{NH}_{2}$ groups toward the negatively charged surface. ${ }^{32}$ For an aqueous solution with $2 \mathrm{mM} \mathrm{DTAB}$ and $1 \mathrm{M}$ urea the two bands are negative, indicating that the urea molecules are pointing with their $\mathrm{C}=\mathrm{O}$ group toward the positively charged surface. The strong dependence of the sign of the vibrational bands of urea on the nature of the surface charge and the lack of any signal from urea for a neutral aqueous surface show that the HD-VSFG response of urea results from an induced orientation of the urea molecules within the electric double layer (EDL) near the surface. Therefore, this response can be described as an electric field induced $\chi_{\text {Urea }}^{(3)}$ effect, similar to the $\chi^{(3)}$ effect that has been observed previously for water molecules at charged interfaces. ${ }^{33-35}$

$$
\begin{aligned}
& E_{\mathrm{VSFG}} \sim \chi_{\mathrm{H}_{2} \mathrm{O}, \text { quad }}^{(2)} N_{\mathrm{H}_{2} \mathrm{O}}+\left(\chi_{\mathrm{H}_{2} \mathrm{O}, \text { dip }}^{(3)} N_{\mathrm{H}_{2} \mathrm{O}}+\chi_{\text {urea,dip }}^{(3)} N_{\text {urea }}\right) \\
& E_{\mathrm{DC} \text {, surf }}
\end{aligned}
$$

Here $E_{\mathrm{VSFG}}$ is the VSFG response and $\chi^{(2)}$ is the secondorder nonlinear susceptibility. $E_{\mathrm{DC} \text {,surf }}$ represents the static electric field within the EDL, and $N_{\mathrm{H}_{2} \mathrm{O}}$ and $N_{\text {urea }}$ represent the number densities of water and urea, respectively. $\chi_{\mathrm{H}_{2} \mathrm{O} \text {,dip }}^{(3)}$ and $\chi_{\text {urea,dip }}^{(3)}$ are the responses resulting from the electric fieldinduced orientation of water and urea molecules within the EDL. We find no indication for the presence of a quadrupolar $\chi^{(2)}$ contribution of urea molecules, suggesting that this contribution is negligible.

To study the properties of urea near aqueous surfaces in more detail, we measured the dependence of the spectra on the urea concentration. In Figure 3, we present the $\operatorname{Im}\left[\chi^{(2)}\right]$ of aqueous (a) SDS and (b) DTAB solutions for different concentrations of urea in a range from 1 to $6 \mathrm{M}$. The concentration of SDS and DTAB are kept constant at $2 \mathrm{mM}$. For all measurement results shown in Figure 3, the response of the water bending mode has been subtracted. For both the SDS and the DTAB solutions, we observe that the absolute amplitudes of the bands at 1660 and $1590 \mathrm{~cm}^{-1}$ increase with increasing urea concentration. This increase reflects an increased amount of oriented urea molecules. To study the concentration dependence of the amplitudes quantitatively, we decompose the responses measured at different concentrations of urea in two Lorentzian bands centered at 1660 and 1590 $\mathrm{cm}^{-1}$. In this decomposition routine, we keep the central frequency and the width of the bands constant and we only allow the amplitude to vary with urea concentration. The resulting fits are shown in parts $a$ and $b$ of Figure 3 with dashed 

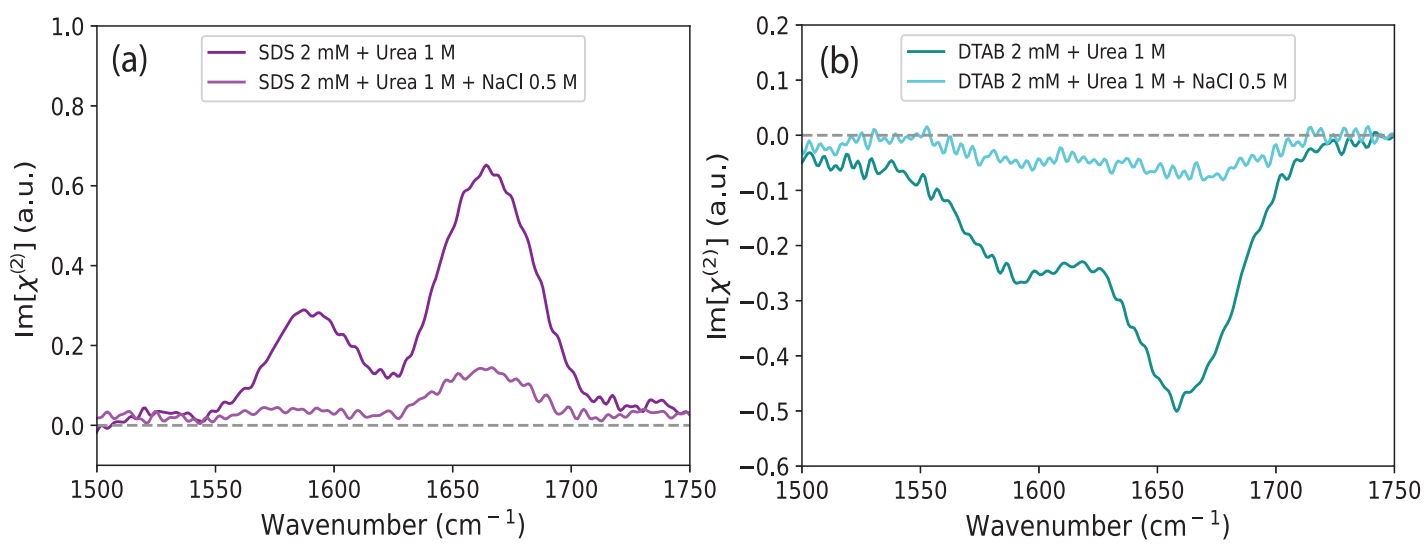

Figure 4. (a) $\operatorname{Im}\left[\chi^{(2)}\right]$ spectra of the $2 \mathrm{mM}$ aqueous SDS solutions with $1 \mathrm{M}$ of urea (dark magenta) and an additional $0.5 \mathrm{M} \mathrm{NaCl}$ added to the solution (magenta). (b) $\operatorname{Im}\left[\chi^{(2)}\right]$ spectra of the $2 \mathrm{mM}$ aqueous DTAB solutions with $1 \mathrm{M}$ of urea (darkcyan) and an additional $0.5 \mathrm{M} \mathrm{NaCl}$ added to the solution (cyan).

lines. The fit results are shown in detail in the Supporting Information (Figures S3 and S4).

Parts $\mathrm{c}$ and $\mathrm{d}$ of Figure 3 show the amplitudes of the two vibrational modes extracted from the fitting procedure as a function of the urea concentration. For both SDS and DTAB, the amplitude of the 1660 and $1590 \mathrm{~cm}^{-1}$ modes first increases proportional to the urea concentration and then shows clear saturation, starting at a urea concentration of $\sim 4 \mathrm{M}$. This saturation behavior can be explained from the dipole-dipole interactions between urea molecules close to the surface. With increasing urea concentration, the electric field experienced by a urea molecule will become increasingly screened by neighboring urea dipoles, thus leading to a decrease of the degree of orientation of all urea molecules.

In Figure 4, we study the effect of adding $0.5 \mathrm{M} \mathrm{NaCl}$ to a solution of $1 \mathrm{M}$ urea and $2 \mathrm{mM}$ SDS (a) and a solution of $1 \mathrm{M}$ urea and of $2 \mathrm{mM} \mathrm{DTAB}$ (b). The spectra shown here are difference spectra of the spectra measured for solutions with and without $1 \mathrm{M}$ of urea, keeping the concentrations of SDS/ $\mathrm{DTAB}$ and $\mathrm{NaClother} \mathrm{components} \mathrm{of} \mathrm{the} \mathrm{solution} \mathrm{the} \mathrm{same,} \mathrm{in}$ order to remove the response from the water bending mode. For SDS and DTAB solutions, the response of the water bending mode is strongly affected by adding $\mathrm{NaCl}^{25}$ We observe that, after adding $\mathrm{NaCl}$ to the solution, the amplitudes of the vibrational modes of urea substantially decrease. This decrease indicates that the net orientation of the urea molecules strongly decrease upon the addition of $\mathrm{Na}^{+}$and $\mathrm{Cl}^{-}$ions. Previous studies of the responses of the $\mathrm{OH}$ stretch band of water and the dipolar contribution of the bending mode of water near charged surfaces showed a similar decrease in intensity upon the addition of sodium chloride. ${ }^{33,36,37}$ This decrease has been explained from the screening of the electric field in the EDL by the added ions, leading to a decrease of the degree of orientation of the water molecules. ${ }^{37,38}$ The observed decrease in the amplitudes of the vibrational bands of urea indicates that a similar effect occurs for urea, i.e., screening of the electric field of the surfactant molecules leads to a decrease of the net orientation of the urea molecules near the surface. The observed decrease in the signal of urea is similar to the one observed for the dipolar response of the bending mode of water. $^{25}$ This observation indicates that the orientation of urea and water molecules in a local electric field are highly correlated, which indicates that urea and water form a collective hydrogen bond network. Application of an electric field induces a net orientation of this collective hydrogen bond network, thus leading to a similar orientational effect on the responses of the urea and water molecules forming the network.

To conclude, we performed heterodyne-detected vibrational sum frequency generation (HD-VSFG) measurements on aqueous urea solutions and of aqueous urea solutions with ionic surfactants. We find that the HD-VSFG spectra of solutions only containing urea do not show characteristic vibrational signatures of urea, showing that urea has no distinct orientation in the region near a neutral aqueous surface. Charging the surface with ionic surfactants leads to the observation of a high-frequency $\left(1660 \mathrm{~cm}^{-1}\right)$ and a lowfrequency $\left(1590 \mathrm{~cm}^{-1}\right)$ band that can be assigned to urea vibrations of mixed $\mathrm{C}=\mathrm{O}$ stretch and $\mathrm{NH}_{2}$ bending character.

The signs and amplitudes of the vibrational bands of urea strongly depend on the sign and amplitude of the surface electric field, which implies that the response of urea is a fieldinduced $\chi_{\text {Urea }}^{(3)}$ effect, similar to the $\chi^{(3)}$ effect observed for the $\mathrm{OH}$ stretch vibration of water and the dipolar contribution of the bending vibration of water at charged aqueous surfaces. The $\chi_{\text {Urea }}^{(3)}$ effect is observed to saturate at a urea concentration of $\sim 4 \mathrm{M}$, probably due to screening of the surface field by the urea molecules. We thus conclude that urea molecules constitute an excellent probe of the interfacial electric field.

Finally, we find that the response of the urea molecules is strongly reduced when the surface electric field is screened by adding $\mathrm{NaCl}$ to the solution, similar to the effect observed for the $\mathrm{OH}$ stretch vibration of water and the dipolar response of the bending vibration of water. This observation shows that the orientations of urea and water molecules in a local electric field are highly correlated, which indicates that urea and water form a collective hydrogen bond network.

For the HD-VSFG measurements we use an amplified Ti:sapphire laser system $(1 \mathrm{kHz}, 35 \mathrm{fs}, 6.5 \mathrm{~mJ} /$ pulse, Coherent "Duo Legend USP") to generate a narrow $800 \mathrm{~nm}$ beam and a tune-able, broadband mid-infrared beam. The two beams are spatially and temporally overlapped at the surface of a gold mirror to generate light at the sum-frequency that serves as a local oscillator (LO-SFG). Before the $800 \mathrm{~nm}$ beam (spolarized), the IR beam (p-polarized), and the LO-SFG signal (s-polarized) are focused on the sample surface, the LO-SFG signal is passed through a silica plate to delay it in time $(\sim 1.6$ ps) with respect to the other beams. The $800 \mathrm{~nm}$ and IR beam 
are generating a VSFG signal at the sample surface. Both the VSFG signal of the sample and the LO-SFG signal are detected with a thermoelectrically cooled charged-coupled device (CCD, Princeton Instruments). From the interference spectrum of the two SFG signals the real (Re) and the imaginary $(\mathrm{Im}) \chi^{(2)}$ spectra can be extracted, providing direct information on the orientation of the vibrational transition dipole moments at the surface, and thus on the absolute orientation of the molecules at the surface. ${ }^{39}$ Additionally, we perform reference experiments with a $z$-cut quartz crystal. In order to obtain high-quality data within the $1500-1750 \mathrm{~cm}^{-1}$ frequency region, we take two independent measurements of $z$ cut quartz with a different orientation of 180 degrees. Thereby we change the phase of our reference measurement exactly by 180 degrees. Upon addition of these two quartz spectra, the interference induced by the SFG signal of the local oscillator and the quartz is removed. The remaining modulation represents the structural noise on the CCD camera induced by an etaloning effect, can now be used as a scaling factor to remove the effect of this modulation from the data. A more detailed explanation of this procedure can be found elsewhere. ${ }^{25}$ For all measurements, we used water from a Millipore Nanopure system (18.2 M $\Omega \mathrm{cm}$, "Simplicity"). Urea, DTAB (dodecyltrimethylammonium bromide), and SDS (sodium dodecyl sulfate) were purchased from Sigma-Aldrich and are used without further purification.

\section{ASSOCIATED CONTENT}

\section{SI Supporting Information}

The Supporting Information is available free of charge at https://pubs.acs.org/doi/10.1021/acs.jpclett.1c03012.

Figure S1, model of the expected phase distortion using the Poisson-Boltzmann equation for a solution with an ionic strength of $2 \mathrm{mM}$; Figure $\mathrm{S} 2, \operatorname{Im}\left[\chi^{(2)}\right]$ spectra of 2 $\mathrm{mM}$ SDS and DTAB solutions with concentrations of 1 and $3 \mathrm{M}$ of deuterated urea in $\mathrm{D}_{2} \mathrm{O}$; Figure S3, spectral decomposition of experimentally obtained $\operatorname{Im}\left[\chi^{(2)}\right]$ spectra of a solution with $2 \mathrm{mM}$ SDS and different urea concentrations; and Figure S4, spectral decomposition of experimentally obtained $\operatorname{Im}\left[\chi^{(2)}\right]$ spectra of a solution with $2 \mathrm{mM}$ DTAB and different urea concentrations (PDF)

\section{AUTHOR INFORMATION}

\section{Corresponding Author}

Huib J. Bakker - Ultrafast Spectroscopy, Amolf, 1098 XG Amsterdam, The Netherlands; 10 orcid.org/0000-00031564-5314; Phone: +31 (0)20 754 7100; Email: H.Bakker@amolf.nl; Fax: +31 (0)20 7547290

\section{Authors}

Carolyn J. Moll - Ultrafast Spectroscopy, Amolf, 1098 XG Amsterdam, The Netherlands; (1) orcid.org/0000-00016041-5898

Jan Versluis - Ultrafast Spectroscopy, Amolf, 1098 XG Amsterdam, The Netherlands

Complete contact information is available at: https://pubs.acs.org/10.1021/acs.jpclett.1c03012

\section{Notes}

The authors declare no competing financial interest.

\section{ACKNOWLEDGMENTS}

This work is part of the research program of The Netherlands Organization for Scientific Research (NWO) and was performed at the research institute AMOLF. This project has received funding from the European Research Council (ERC) under the European Union's Horizon 2020 research and innovation program (Grant Agreement No. 694386)

\section{REFERENCES}

(1) Frank, H. S.; Franks, F. Structural approach to the solvent power of water for hydrocarbons; Urea as a structure breaker. J. Chem. Phys. 1968, 48, 4746-4757.

(2) Rezus, Y. L. A.; Bakker, H. J. Effect of urea on the structural dynamics of water. Proc. Natl. Acad. Sci. U. S. A. 2006, 103, 1841718420.

(3) Bruning, W.; Holtzer, A. The effect of urea on hydrophobic bonds: The critical micelle concentration of $\mathrm{n}$-dodecyltrimethylammonium bromide in aqueous solutions of urea. J. Am. Chem. Soc. 1961, 83, 4865-4866.

(4) Åstrand, P. O.; Wallqvist, A.; Karlström, G. Molecular dynamics simulations of $2 \mathrm{~m}$ aqueous urea solutions. J. Phys. Chem. 1994, 98, 8224-8233.

(5) Brandts, J. F.; Hunt, L. The Thermodynamics of Protein Denaturation. III. The Denaturation of Ribonuclease in Water and in Aqueous Urea and Aqueous Ethanol Mixtures. J. Am. Chem. Soc. 1967, 89, 4826-4838.

(6) Sagle, L. B.; Zhang, Y.; Litosh, V. A.; Chen, X.; Cho, Y.; Cremer, P. S. Investigating the hydrogen-bonding model of urea denaturation. J. Am. Chem. Soc. 2009, 131, 9304-9310.

(7) Rossky, P. J. Protein denaturation by urea: Slash and bond. Proc. Natl. Acad. Sci. U. S. A. 2008, 105, 16825-16826.

(8) Auton, M.; Bolen, D. W. Predicting the energetics of osmolyteinduced protein folding unfolding. Proc. Natl. Acad. Sci. U. S. A. 2005, 102, 15065-15068.

(9) Kaatze, U.; Gerke, H.; Pottel, R. Dielectric relaxation in aqueous solutions of urea and some of its derivatives. J. Phys. Chem. 1986, 90, 5464-5469.

(10) Soper, A. K.; Castner, E. W.; Luzar, A. Impact of urea on water structure: A clue to its properties as a denaturant? Biophys. Chem. 2003, 105, 649-666.

(11) Finer, E. G.; Franks, F.; Tait, M. J. Nuclear Magnetic Resonance Studies of Aqueous Urea Solutions. J. Am. Chem. Soc. 1972, 94, 4424-4429.

(12) Idrissi, A.; Gerard, M.; Damay, P.; Kiselev, M.; Puhovsky, Y.; Cinar, E.; Lagant, P.; Vergoten, G. The effect of urea on the structure of water: A molecular dynamics simulation. J. Phys. Chem. B 2010, $114,4731-4738$.

(13) Walrafen, G. E. Roman spectral studies of the effects of urea and sucrose on water structure. J. Chem. Phys. 1966, 44, 3726-3727.

(14) Funkner, S.; Havenith, M.; Schwaab, G. Urea, a Structure Breaker? Answers from THz Absorption Spectroscopy. J. Phys. Chem. B 2012, 116, 13374-13380.

(15) Shimizu, A.; Fumino, K.; Yukiyasu, K.; Taniguchi, Y. NMR studies on dynamic behavior of water molecule in aqueous denaturant solutions at $25{ }^{\circ} \mathrm{C}$ : Effects of guanidine hydrochloride, urea and alkylated ureas. J. Mol. Liq. 2000, 85, 269-278.

(16) Halonen, S.; Kangas, T.; Haataja, M.; Lassi, U. Urea-WaterSolution Properties: Density, Viscosity, and Surface Tension in an Under-Saturated Solution. Emission Contr. Sci. Technol. 2017, 3, 161170

(17) Terzis, A.; Sauer, E.; Yang, G.; Groß, J.; Weigand, B. Characterisation of acid-base surface free energy components of urea-water solutions. Colloids Surf., A 2018, 538, 774-780.

(18) Stumpe, M. C.; Grubmüller, H. Aqueous urea solutions: Structure, energetics, and urea aggregation. J. Phys. Chem. B 2007, $111,6220-6228$. 
(19) Bandyopadhyay, D.; Mohan, S.; Ghosh, S. K.; Choudhury, N. Molecular dynamics simulation of aqueous urea solution: Is urea a structure breaker? J. Phys. Chem. B 2014, 118, 11757-11768.

(20) Breslow, R.; Guo, T. Surface tension measurements show that chaotropic salting-in denaturants are not just water-structure breakers. Proc. Natl. Acad. Sci. U. S. A. 1990, 87, 167-169.

(21) Strazdaite, S.; Meister, K.; Bakker, H. J. Orientation of polar molecules near charged protein interfaces. Phys. Chem. Chem. Phys. 2016, 18, 7414-7418.

(22) Chen, X.; Sagle, L. B.; Cremer, P. S. Urea Orientation at Protein Surfaces. J. Am. Chem. Soc. 2007, 129, 15104-15105.

(23) Kundu, A.; Tanaka, S.; Ishiyama, T.; Ahmed, M.; Inoue, K. I.; Nihonyanagi, S.; Sawai, H.; Yamaguchi, S.; Morita, A.; Tahara, T. Bend Vibration of Surface Water Investigated by HeterodyneDetected Sum Frequency Generation and Theoretical Study: Dominant Role of Quadrupole. J. Phys. Chem. Lett. 2016, 7, 25972601.

(24) Ahmed, M.; Nihonyanagi, S.; Kundu, A.; Yamaguchi, S.; Tahara, T. Resolving the Controversy over Dipole versus Quadrupole Mechanism of Bend Vibration of Water in Vibrational Sum Frequency Generation Spectra. J. Phys. Chem. Lett. 2020, 11, 9123-9130.

(25) Moll, C. J.; Versluis, J.; Bakker, H. J. Direct Evidence for a Surface and Bulk Specific Response in the Sum-Frequency Generation Spectrum of the Water Bend Vibration. Phys. Rev. Lett. 2021, 127, 116001.

(26) Gragson, D. E.; Richmond, G. L. Investigations of the Structure and Hydrogen Bonding of Water Molecules at Liquid Surfaces by Vibrational Sum Frequency Spectroscopy. J. Phys. Chem. B 1998, 102, 3847-3861.

(27) Ohno, P. E.; Wang, H. F.; Paesani, F.; Skinner, J. L.; Geiger, F. M. Second-Order Vibrational Lineshapes from the Air/Water Interface. J. Phys. Chem. A 2018, 122, 4457-4464.

(28) Ohno, P. E.; Wang, H. F.; Geiger, F. M. Second-order spectral lineshapes from charged interfaces. Nat. Commun. 2017, 8, 1-9.

(29) Puranik, P. G.; Sirdeshmukh, L. Vibrational spectra, force constants and meanamplitudes of vibrations of urea and deuterated urea. Proc. - Indian Acad. Sci., Sect. A 1968, 67, 99-108.

(30) Stewart, J. E. Infrared Absorption Spectra of Urea etc. J. Chem. Phys. 1957, 26, 248-254.

(31) Carr, J. K.; Buchanan, L. E.; Schmidt, J. R.; Zanni, M. T.; Skinner, J. L. Structure and Dynamics of Urea/Water Mixtures Investigated by Vibrational Spectroscopy and Molecular Dynamics Simulation. J. Phys. Chem. B 2013, 117, 13291-13300.

(32) Okuno, M.; Yamada, S.; Ohto, T.; Tada, H.; Nakanishi, W.; Ariga, K.; Ishibashi, T. A. Hydrogen Bonds and Molecular Orientations of Supramolecular Structure between Barbituric Acid and Melamine Derivative at the Air/Water Interface Revealed by Heterodyne-Detected Vibrational Sum Frequency Generation Spectroscopy. J. Phys. Chem. Lett. 2020, 11, 2422-2429.

(33) Nihonyanagi, S.; Yamaguchi, S.; Tahara, T. Direct evidence for orientational flip-flop of water molecules at charged interfaces: A heterodyne-detected vibrational sum frequency generation study. J. Chem. Phys. 2009, 130, 204704.

(34) Morita, A. Theory of Sum Frequency Generation Spectroscopy; Springer: Singapore, 2018.

(35) Mondal, J. A.; Nihonyanagi, S.; Yamaguchi, S.; Tahara, T. Structure and orientation of water at charged lipid monolayer/water interfaces probed by heterodyne-detected vibrational sum frequency generation spectroscopy. J. Am. Chem. Soc. 2010, 132, 10656-10657.

(36) Tian, C. S.; Shen, Y. R. Structure and charging of hydrophobic material/water interfaces studied by phase-sensitive sum-frequency vibrational spectroscopy. Proc. Natl. Acad. Sci. U. S. A. 2009, 106, $15148-15153$.

(37) Sartin, M. M.; Sung, W.; Nihonyanagi, S.; Tahara, T. Molecular mechanism of charge inversion revealed by polar orientation of interfacial water molecules: A heterodyne-detected vibrational sum frequency generation study. J. Chem. Phys. 2018, 149, 024703.
(38) Nihonyanagi, S.; Yamaguchi, S.; Tahara, T. Counterion Effect on Interfacial Water at Charged Interfaces and Its Relevance to the Hofmeister Series. J. Am. Chem. Soc. 2014, 136, 6155-6158.

(39) Shen, Y. R. Phase-Sensitive Sum-Frequency Spectroscopy. Annu. Rev. Phys. Chem. 2013, 64, 129. 